\title{
The Dynamics Analysis of Multiscale Eigenelement Method on Periodic Composite Materials
}

\author{
Wu Mengmeng ${ }^{1, *}$, Jia Ruiyu ${ }^{2}$ \\ ${ }^{1}$ College of Science, Naval University of Engineering, Wuhan, China \\ ${ }^{2}$ Municipal and Traffic Engineering Design Institute, Changjiang Institute of Survey Planning Design and Research, Wuhan, China
}

\section{Email address:}

mengmengwu1017@163.com(Wu Mengmeng),cjwjry@163.com (Jia Ruiyu)

*Corresponding author

\section{To cite this article:}

Wu Mengmeng, Jia Ruiyu. The Dynamics Analysis of Multiscale Eigenelement Method on Periodic Composite Materials. Science Discovery. Vol. 5, No. 6, 2017, pp. 404-409. doi: 10.11648/j.sd.20170506.11

Received: August 2, 2017; Accepted: September 25, 2017; Published: October 27, 2017

\begin{abstract}
As the composite materials and structures are widely used in aerospace, it is necessary to develop more precise and higher efficient methods to predict the mechanical properties and behaviors of the composites for application. The multiscale eigenelement method (MEM) can be implemented to analysize the periodic composite materials. This paper has reserched the MEM accuracy and mechanical behavior, and deduced the dynamics analytical solution of periodic composite materials. Compared the influence to the accuracy of different model numbers. As for the problems of multiscale eigenelement method, we put forward unit cell modal method. The results of the numerical calculation indicate the effectiveness and feasibility of the unit cell modal method.
\end{abstract}

Keywords: Composite Materials, Multi-Scale Method, Eigen-Element Method, Unit-Cell

\section{周期结构多尺度特征单元法动力学分析}

吴蒙蒙 ${ }^{1, *}$, 贾瑞雨 ${ }^{2}$

${ }^{1}$ 理学院, 海军工程大学, 武汉, 中国

2市政与交通工程设计院, 长江勘测规划设计研究有限责任公司, 武汉, 中国

邮箱

mengmengwu1017@163.com(吴蒙蒙), cjwjry@163.com（贾瑞雨）

摘要：随着新型复合材料的研究、设计及应用的日益增长, 发展高精度高效率的分析方法对其进行理论研究和性能预 估, 是推动航空航天技术进一步发展的基础性课题。多尺度特征单元方法 (MEM) 是分析周期复合材料结构力学行为 的常用方法, 本文针对MEM的精度和力学含义进行研究, 推导了周期复合材料结构的多尺度特征单元方法动力学解析 解, 比较了不同的单胞模态数对计算结果精度的影响, 并以一维梁结构为例进行了数值计算, 数值计算结果与传统有 限元计算结果比较吻合，证明了多尺度特征单元方法的有效性和可行性。

关键词：复合材料，多尺度，特征单元，单胞 


\section{1. 引言}

对于周期复合材料结构问题, 计算其力学性能的多尺 度方法有很多, 本文介绍的多尺度特征单元方法 (MEM) 提高多了尺度方法的实用性, 同时兼顾精度和效率。并且 从力学角度对MEM的意义及其应用问题进行研究, 多尺 度特征单元方法(Multiscale eigenelement method, 简记为 MEM)是基于特征向量展开技术，根据单胞自平衡原理找 到宏观尺度与细观尺度之间的关系, 得到单胞的形函数, 再用有限元方法求解 $[1,2]$ 。多尺度特征单元方法不同于其 他多尺度方法之处在于, 求解单胞问题时无需引入外部约 束以及载荷, 保证了边界的松弛性, 直接由单胞自平衡方 程 [3，4]得到了单胞宏观结点位移和细观结点位移之间的 关系。由此可知, 多尺度特征单元方法巧妙地避免了复杂 边界的求解, 直接由单胞自平衡方程得到了单胞宏观结点 位移和细观结点位移之间的关系, 提高了计算结果的精度 和效率。而在特征单元构造中, 形函数 $\mathrm{N}$ 的构造是核心问 题, 其可以反映单胞更多的细观信息。

为了更加清楚地描述MEM的精度和力学含义, 本文 选择了一维周期复合材料结构为研究对象。

\section{2. 形函数的构造}

如图1所示多尺度特征单元单胞通常要保留所有细观 单元在单胞边界的结点, 称之为特征单元结点 $[5,6]$ 。

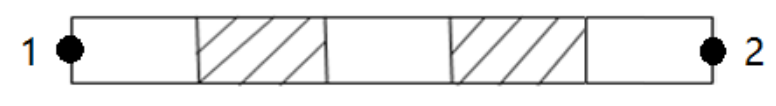

图1 特征单元单胞模型。

在特征单元构造中, 形函数 $N$ 的构造决定了方法的精 度, 多尺度特征单元方法直接由单胞自平衡方程得到了单 胞宏观结点位移和细观结点位移之间的关系得到单胞的 形函数。首先将单胞进行有限元离散, 在没有外部载荷情 况下, 单胞的自平衡方程为:

$$
\boldsymbol{K u}=\mathbf{0}
$$

式中: $K$ 表示由细网格有限元法组装得到单胞的总刚 度矩阵, 依据单胞的复杂程度划分网格, 包含材料突变信 息。MEM解决单胞问题不用求解具体数值, 通过式 (1) 得到宏观结点位移与细观结点位移之间的关系 [7,8]。分离 式（1）宏观和细观尺度信息, 可以得到:

$$
\left[\begin{array}{ll}
\boldsymbol{K}_{\mathrm{ee}} & \boldsymbol{K}_{\mathrm{ei}} \\
\boldsymbol{K}_{\mathrm{ie}} & \boldsymbol{K}_{\mathrm{ii}}
\end{array}\right]\left[\begin{array}{c}
\boldsymbol{u}_{\mathrm{e}} \\
\boldsymbol{u}_{\mathrm{i}}
\end{array}\right]=\left[\begin{array}{l}
\boldsymbol{0} \\
\boldsymbol{0}
\end{array}\right]
$$

式中: 下标“e”表示单胞边界结点, 下标“i”表示单胞 内部结点, $\boldsymbol{u}_{i}$ 为细观变量, 则由矩阵第二行得:

$$
\boldsymbol{u}_{\mathrm{i}}=-\boldsymbol{K}_{\mathrm{ii}}^{-1} \boldsymbol{K}_{\mathrm{ie}} \boldsymbol{u}_{\mathrm{e}}
$$

用形函数 $N$ 和宏观变量 $\boldsymbol{u}_{\mathrm{e}}$ 表示细观模型下的位移变 量 $u$, 即:

$$
u=N u_{e}
$$

将式(3)代入到(4), 则有:

$$
\boldsymbol{u}=\left[\begin{array}{c}
\boldsymbol{u}_{\mathrm{e}} \\
\boldsymbol{u}_{\mathrm{i}}
\end{array}\right]=\left[\begin{array}{c}
\boldsymbol{I} \\
-\boldsymbol{K}_{\mathrm{ii}}^{-1} \boldsymbol{K}_{\mathrm{ie}}
\end{array}\right] \boldsymbol{u}_{\mathrm{e}}
$$

式中: $\boldsymbol{u}_{\mathrm{e}}$ 是MEM的特征单元结点位移。根据式 (4) 和式 (5) 得到MEM的形函数为:

$$
\boldsymbol{N}=\left[\begin{array}{c}
\boldsymbol{I} \\
-\boldsymbol{K}_{\mathrm{ii}}^{-1} \boldsymbol{K}_{\mathrm{ie}}
\end{array}\right]
$$

$N$ 中每一列的元素代表宏观结点形函数在各细观结点 的值。根据单胞总势能泛函和动能系数泛函与有限元法相 等, 即可求解得到特征单元的刚度矩阵 $K_{\mathrm{G}}$, 载荷列向量 $F_{\mathrm{G}}$ 以及质量矩阵 $M_{\mathrm{G}}$ 。

$$
\begin{aligned}
\Pi & =\frac{1}{2} \boldsymbol{u}^{\mathrm{T}} \boldsymbol{K} \boldsymbol{u}-\boldsymbol{u}^{\mathrm{T}} \boldsymbol{f}=\frac{1}{2} \boldsymbol{u}_{\mathrm{G}}^{\mathrm{T}} \boldsymbol{N}^{\mathrm{T}} \boldsymbol{K} \boldsymbol{N} \boldsymbol{u}_{\mathrm{G}}-\boldsymbol{u}_{\mathrm{G}}^{\mathrm{T}} \boldsymbol{N}^{\mathrm{T}} \boldsymbol{f} \\
& =\frac{1}{2} \boldsymbol{u}_{\mathrm{G}}^{\mathrm{T}} \boldsymbol{K}_{\mathrm{G}} \boldsymbol{u}_{\mathrm{G}}-\boldsymbol{u}_{\mathrm{G}}^{\mathrm{T}} \boldsymbol{f}_{\mathrm{G}} \\
T_{0} & =\frac{1}{2} \boldsymbol{u}^{\mathrm{T}} \boldsymbol{M} \boldsymbol{u}=\frac{1}{2} \boldsymbol{u}_{\mathrm{G}}^{\mathrm{T}} \boldsymbol{N}^{\mathrm{T}} \boldsymbol{M} \boldsymbol{N} \boldsymbol{u}_{\mathrm{G}}=\frac{1}{2} \boldsymbol{u}_{\mathrm{G}}^{\mathrm{T}} \boldsymbol{M}_{\mathrm{G}} \boldsymbol{u}_{\mathrm{G}}
\end{aligned}
$$

上面公式是不同特征单元方法的通用数学基础。式中, $u_{\mathrm{G}}$ 是MEM的特征单元结点位移, $K_{\mathrm{G}}$ 可以反映单胞的细观 材料属性和几何特征。值得一提的是, 由于特征单元形函 数 $N$ 直接由刚度矩阵 $K$ 得出, $K_{\mathrm{G}}$ 也不需要通过数值积分得 到, 即:

$$
\begin{gathered}
\boldsymbol{K}_{\mathrm{G}}=\boldsymbol{K}_{\mathrm{ee}}-\boldsymbol{c} \boldsymbol{K}_{\mathrm{ei}}, \quad \boldsymbol{c}=\boldsymbol{K}_{\mathrm{ei}} \boldsymbol{K}_{\mathrm{ii}}^{-1} \\
\boldsymbol{M}_{\mathrm{G}}=\boldsymbol{M}_{\mathrm{ee}}-\boldsymbol{c} \boldsymbol{M}_{\mathrm{ie}}-\left(\boldsymbol{c} \boldsymbol{M}_{\mathrm{ie}}\right)^{\mathrm{T}}+\boldsymbol{c} \boldsymbol{M}_{\mathrm{ii}} \boldsymbol{c}^{\mathrm{T}} \\
\boldsymbol{f}_{\mathrm{G}}=\boldsymbol{N}^{\mathrm{T}} \boldsymbol{f}=\boldsymbol{f}_{\mathrm{e}}-\boldsymbol{c} \boldsymbol{f}_{\mathrm{i}}
\end{gathered}
$$

将上面求得的宏观单元刚度矩阵, 质量矩阵, 以及载 荷列向量, 利用有限元方法组装, 得到:

$$
\boldsymbol{K}_{\mathrm{C}}=\mathbf{A}_{i=1}^{\mathrm{m}} \boldsymbol{K}_{\mathrm{G}}, \boldsymbol{M}_{\mathrm{C}}=\mathbf{A}_{i=1}^{\mathrm{m}} \boldsymbol{M}_{\mathrm{G}}, \boldsymbol{F}_{\mathrm{C}}=\mathbf{A}_{i=1}^{\mathrm{m}} \boldsymbol{F}_{\mathrm{G}}
$$

其中, 定义的算子 $\mathrm{A}$ 代表组装各单元矩阵; $K_{\mathrm{C}}, M_{\mathrm{C}}$, $F_{\mathrm{C}}$ 代表整体结构的刚度矩阵质量矩阵和载荷向量, 代入广 义的特征值方程[9-13]得:

$$
\boldsymbol{K} \boldsymbol{\varphi}_{i}=\omega_{i}^{2} \boldsymbol{M} \boldsymbol{\varphi}_{i}
$$

可以求出复合材料的各阶频率和模态。 $\varphi_{i}$ 表示第 $i$ 介 模态, 对应的频率为 $\omega_{i}$ 。

\section{3. 多尺度特征单元方法动力学推导}

为了提高MEM在计算动力学问题的精度, 本文在原 单胞形函数基础上构造出新的特征单元形函数, 新的单胞 的形函数和结点位移向量的构造方式可以表示为: 


$$
\begin{gathered}
\boldsymbol{N}_{\text {new }}=\left[\begin{array}{ll}
\boldsymbol{N} & \boldsymbol{N}_{m}
\end{array}\right] \\
\boldsymbol{u}=\boldsymbol{N} \boldsymbol{u}_{\mathrm{e}}+\boldsymbol{N}_{m} \boldsymbol{u}_{m}=\left[\begin{array}{ll}
N & \boldsymbol{N}_{m}
\end{array}\right]\left[\begin{array}{l}
\boldsymbol{u}_{\mathrm{e}} \\
\boldsymbol{u}_{m}
\end{array}\right]
\end{gathered}
$$

式中, $N$ 与原特征单元形函数构造方法相同, $\boldsymbol{u}_{\mathrm{e}}$ 为单 胞特征结点对应的位移, $N_{m}$ 为由单胞前 $\mathrm{m}$ 阶模态构成的模 态形函数, $\boldsymbol{u}_{m}$ 为其对应的广义坐标。根据线性代数知识, 任何一组位移向量可由相同维数的向量空间线性组合构成, 而单胞的各阶模态恰可构成一组向量空间，因此取单胞的 低阶模态可很好的起到描述单胞内部位移和模态的作用。

矩阵 $\boldsymbol{N}_{m}$ 可写作下面形式:

$$
N_{\mathrm{m}}=\left[\begin{array}{llll}
\varphi_{1} & \varphi_{2} & \cdots & \varphi_{m}
\end{array}\right]
$$

其中 $\boldsymbol{\varphi}_{i},(i=1,2, \cdots, m)$ 可以由下面的广义特征值方程 求解:

$$
\boldsymbol{K} \boldsymbol{\varphi}_{i}=\omega_{i}^{2} \boldsymbol{M} \boldsymbol{\varphi}_{i}
$$

边界条件为:

$$
\boldsymbol{\varphi}_{i}=\boldsymbol{0}, \text { on } \Omega
$$

式(16)中的矩阵 $\boldsymbol{K}$ 和 $\boldsymbol{M}$ 是单胞的细网格刚度矩阵和 质量矩阵。

将新构造的形函数 $N_{n e w}=\left[\begin{array}{ll}N & N_{m}\end{array}\right]$ 代入到特征单元 刚度矩阵、质量矩阵和载荷矩阵中可以得到:

$$
\begin{aligned}
& \boldsymbol{K}_{\text {Gew }}=\boldsymbol{N}_{\text {new }}^{\mathrm{T}} \boldsymbol{K} \boldsymbol{N}_{\mathrm{naw}}=\left[\begin{array}{ll}
\boldsymbol{N}^{\mathrm{T}} \boldsymbol{K} \boldsymbol{N} & \boldsymbol{N}^{\mathrm{T}} \boldsymbol{K} \boldsymbol{N}_{m} \\
\boldsymbol{N}_{m}^{\mathrm{T}} \boldsymbol{K} \boldsymbol{N} & \boldsymbol{N}_{m}^{\mathrm{T}} \boldsymbol{K} \boldsymbol{N}_{m}
\end{array}\right] \\
& \boldsymbol{M}_{\text {chew }}=\boldsymbol{N}_{\text {new }}^{\mathrm{T}} \boldsymbol{M} \boldsymbol{N}_{\text {new }}=\left[\begin{array}{ll}
\boldsymbol{N}^{\mathrm{T}} \boldsymbol{M} & \boldsymbol{N}^{\mathrm{T}} \boldsymbol{M} \boldsymbol{N}_{m} \\
\boldsymbol{N}_{m}^{\mathrm{T}} \boldsymbol{M} & \boldsymbol{N}_{m}^{\mathrm{T}} \boldsymbol{M} \boldsymbol{N}_{m}
\end{array}\right] \\
& \boldsymbol{F}_{\text {Gnew }}=\boldsymbol{N}_{\text {new }}^{\mathrm{T}} \boldsymbol{f}=\left[\begin{array}{c}
\boldsymbol{N}^{\mathrm{T}} \boldsymbol{f} \\
\boldsymbol{N}_{m}^{\mathrm{T}} \boldsymbol{f}
\end{array}\right]
\end{aligned}
$$

式中: $\boldsymbol{M}=\left[\begin{array}{cc}\boldsymbol{M}_{e e} & \boldsymbol{M}_{e i} \\ \boldsymbol{M}_{i e} & \boldsymbol{M}_{i i}\end{array}\right] \boldsymbol{K}=\left[\begin{array}{cc}\boldsymbol{K}_{e e} & \boldsymbol{K}_{e i} \\ \boldsymbol{K}_{i e} & \boldsymbol{K}_{i i}\end{array}\right]$

此时, $K_{G}$ 的斜对角子矩阵等于零, 即 $N$ 和 $N_{m}$ 对于 $K$ 是正交的。其正交性证明过如下:

$$
\begin{aligned}
& \boldsymbol{K N}=\left[\begin{array}{cc}
\boldsymbol{K}_{\mathrm{ee}} & \boldsymbol{K}_{\mathrm{ei}} \\
\boldsymbol{K}_{\mathrm{ei}} & \boldsymbol{K}_{\mathrm{ii}}
\end{array}\right]\left[\begin{array}{c}
\boldsymbol{I} \\
-\boldsymbol{K}_{\mathrm{ii}}^{-1} K_{\mathrm{ie}}
\end{array}\right]=\left[\begin{array}{c}
\boldsymbol{K}_{\mathrm{ee}}-K_{\mathrm{ei}} K_{\mathrm{ii}} K_{\mathrm{ie}} \\
\boldsymbol{K}_{\mathrm{ie}}-\boldsymbol{K}_{\mathrm{ie}}
\end{array}\right]=\left[\begin{array}{c}
\tilde{K}_{\mathrm{ee}} \\
\boldsymbol{0}
\end{array}\right] \\
& N_{m}^{\mathrm{T}} \boldsymbol{K N}=N_{m}^{\mathrm{T}}\left[\begin{array}{c}
\tilde{K}_{\mathrm{ee}} \\
0
\end{array}\right]=\left[\begin{array}{c}
N_{m \mathrm{e}} \\
N_{m i}
\end{array}\right]\left[\begin{array}{c}
\tilde{K}_{\mathrm{ee}} \\
0
\end{array}\right]=\left[\begin{array}{ll}
0 & N_{n i}^{\mathrm{T}} \mathrm{T}
\end{array}\right]\left[\begin{array}{c}
\tilde{K}_{\mathrm{ee}} \\
0
\end{array}\right][0]
\end{aligned}
$$

其中, $\boldsymbol{N}_{m e}$ 和 $\boldsymbol{N}_{m i}$ 是 $\boldsymbol{N}_{m}$ 的两个子矩阵, 分别对应各阶 模态形函数在边界节点和内部节点的取值。因此, 式 (18) 化为:

$$
\boldsymbol{K}_{\text {Ginew }}=\left[\begin{array}{cc}
\boldsymbol{N}^{\mathrm{T}} \boldsymbol{K} \boldsymbol{N} & \boldsymbol{0} \\
\boldsymbol{0} & \boldsymbol{N}_{m}^{\mathrm{T}} \boldsymbol{K} \boldsymbol{N}_{m}
\end{array}\right]
$$

将新的刚度矩阵和质量矩阵代入式(16)得到新的广义 特征值方程:

$$
\begin{gathered}
\boldsymbol{K}_{\text {Gnew }} \Phi_{0}=\lambda \boldsymbol{M}_{\text {Gnew }} \Phi_{0} \\
{\left[\begin{array}{cc}
\boldsymbol{N}^{T} \boldsymbol{K} \boldsymbol{N} & \boldsymbol{N}^{T} \boldsymbol{K} \boldsymbol{N}_{\boldsymbol{m}} \\
\boldsymbol{N}_{\boldsymbol{m}}^{T} \boldsymbol{K N} & \boldsymbol{N}_{\boldsymbol{m}}^{T} \boldsymbol{K} \boldsymbol{N}_{\boldsymbol{m}}
\end{array}\right] \boldsymbol{\Phi}_{o}=\lambda\left[\begin{array}{cc}
\boldsymbol{M}_{\boldsymbol{z}} & \boldsymbol{N}^{T} \boldsymbol{M} \boldsymbol{N}_{\boldsymbol{m}} \\
\boldsymbol{N}_{\boldsymbol{m}}^{T} \boldsymbol{M N} & \boldsymbol{M}_{\boldsymbol{m}}
\end{array}\right] \boldsymbol{\Phi}_{o}}
\end{gathered}
$$

把求得的 $(n+m)$ 个特征向量也就是 $(n+m)$ 个模态 按列排列, 组成一个矩阵 $\boldsymbol{\Phi}_{\boldsymbol{o}}$, 即:

$$
\boldsymbol{\Phi}_{\boldsymbol{o}}=\left[\begin{array}{ll}
\boldsymbol{\varphi}_{e((n+m) \times n)} & \boldsymbol{\varphi}_{m((n+m) \times m)}
\end{array}\right]_{((n+m) \times(n+m))}
$$

式中, $n$ 是单胞边界节点数, $m$ 是添加的单胞低阶模态 数。

由式(24)知, 向量 $\boldsymbol{\Phi}_{0}$ 关于 $\boldsymbol{K}_{\mathrm{Gnew}}, \boldsymbol{M}_{\mathrm{Gnew}}$ 正交, 即:

$$
\boldsymbol{\varphi}_{0 i}^{T} \boldsymbol{K}_{\text {Gnew }} \boldsymbol{\varphi}_{0 j}=0, \boldsymbol{\varphi}_{0 i}^{T} \boldsymbol{M}_{\text {Gnew }} \boldsymbol{\varphi}_{0 j}=0, i \neq j
$$

由于模态是相互正交、线性无关的, 因此模态矩阵 $\boldsymbol{\Phi}_{\boldsymbol{o}}$ 一定是满秩的, 即 $\boldsymbol{\Phi}_{\boldsymbol{o}}$ 可逆, 由此得修正后每个单胞的模 态可通过形函数计算得到:

$$
\begin{aligned}
& \Phi_{h \times(n+m)}=N_{\text {new }} \Phi_{0} \\
& =\left[\begin{array}{ll}
N & N_{\mathrm{m}}
\end{array}\right]_{k \times(n+m)}\left[\begin{array}{ll}
\varphi_{e((n+m) \times n)} & \varphi_{m((n+m) \times m)}
\end{array}\right]_{((n+m) \times(n+m))}
\end{aligned}
$$

由式（26）得:

$$
\begin{aligned}
& \boldsymbol{\varphi}_{0 i}^{T} \boldsymbol{N}_{\text {new }}^{T} \boldsymbol{K} \boldsymbol{N}_{\text {new }} \boldsymbol{\varphi}_{0 j}=0, \boldsymbol{\varphi}_{0 i}^{T} \boldsymbol{N}_{\text {new }}^{T} \boldsymbol{M} \boldsymbol{N}_{\text {new }} \boldsymbol{\varphi}_{0 j}=0, i \neq j \\
& \Rightarrow \boldsymbol{\varphi}_{i}^{T} \boldsymbol{K} \boldsymbol{\varphi}_{j}=0, \boldsymbol{\varphi}_{i}^{T} \boldsymbol{M} \boldsymbol{\varphi}_{j}=0, i \neq j
\end{aligned}
$$

所以, $\boldsymbol{\Phi}_{k \times(n+m)}$ 是关于 $\boldsymbol{K}, \boldsymbol{M}$ 正交的矩阵。 $k$ 是单胞 细网格节点数。

组装后, 结构的模态为:

$$
\boldsymbol{\Phi}_{Z}=\left[\begin{array}{llll}
\boldsymbol{\varphi}_{z 1} & \boldsymbol{\varphi}_{z 2} & \ldots & \boldsymbol{\varphi}_{z(n+m)}
\end{array}\right]_{(k \rtimes(n+m))}
$$

式中, $k$ 为整体结构所有结点数, $n+m$ 为单胞的边界 节点与添加的模态向量个数之和, 并且 $n+m<k$ 。

由式 (29) 可知, $\boldsymbol{\Phi}_{Z}$ 是关于 $\boldsymbol{K}_{Z}, \boldsymbol{M}_{Z}$ 正交的矩阵。 $\boldsymbol{K}_{Z}, \boldsymbol{M}_{Z}$ 是由 $\boldsymbol{K}, \boldsymbol{M}$ 组装成的整体细观有限元刚度矩阵 和质量矩阵。

根据模态正交性，容易证明模态之间是线性无关的， 也就是说, $n$ 维系统的 $n$ 个模态构成 $n$ 维向量空间的一组正 交基, 于是该 $n$ 维空间的任意一个向量 $u$ 都可以用这组正交 基来表示。

设结构的响应为:

$$
\boldsymbol{u}_{k \times 1}=\boldsymbol{\Phi}_{Z(k \times(n+m))} \boldsymbol{q}_{(n+m) \times 1}
$$




$$
\begin{aligned}
& \boldsymbol{\Phi}_{Z}^{\top} \boldsymbol{M}_{Z} \boldsymbol{u}_{k \times 1}=\boldsymbol{\Phi}_{Z}^{\top} \boldsymbol{M}_{Z} \boldsymbol{\Phi}_{Z(k \times(n+m))} \boldsymbol{q}_{(n+m) \times 1} \\
& \Rightarrow \boldsymbol{\Phi}_{Z}^{\top} \boldsymbol{M}_{Z} \boldsymbol{u}_{k \times 1}=\boldsymbol{M}_{P Z} \boldsymbol{q}_{(n+m) \times 1} \\
& \Rightarrow\left(\boldsymbol{M}_{P Z}\right)^{-1} \boldsymbol{\Phi}_{Z}^{\top} \boldsymbol{M}_{Z} \boldsymbol{u}_{k \times 1}=\boldsymbol{q}_{(n+m) \times 1}
\end{aligned}
$$

动力学基本方程为:

$$
\boldsymbol{M}_{k \times k} \ddot{\boldsymbol{u}}+\boldsymbol{K}_{k \times k} \boldsymbol{u}=\mathbf{0}
$$

将式（31）代入式(32)得:

$$
\begin{aligned}
& \boldsymbol{M}_{k \times k} \boldsymbol{\Phi}_{(x\langle n+m)} \ddot{\boldsymbol{q}}_{(n+m) \times 1}+\boldsymbol{K}_{k \times k} \boldsymbol{\Phi}_{(x \backslash n+m)} \boldsymbol{q}_{(n+m) \times 1}=\mathbf{0} \\
& \left(\boldsymbol{\Phi}_{k \times(n+m)}\right)^{T} \boldsymbol{M} \boldsymbol{\Phi}_{k \times(n+m)} \ddot{\boldsymbol{g}}_{(n+m) \times 1}+\boldsymbol{\Phi}_{k \times(n+m)}^{T} \boldsymbol{K} \boldsymbol{\Phi}_{k \times(n+m)} \boldsymbol{q}_{(n+m) \times 1}=\boldsymbol{0} \\
& \overline{\boldsymbol{M}}_{(n+m) \times(n+m)} \ddot{\boldsymbol{q}}_{(n+m) \times 1}+\overline{\boldsymbol{K}}_{(n+m) \times(n+m)} \boldsymbol{q}_{(n+m) \times 1}=\boldsymbol{0}
\end{aligned}
$$

设 $\boldsymbol{u}, \boldsymbol{v}, \boldsymbol{a}$ 的初始条件为:

$$
\begin{aligned}
\boldsymbol{u}_{0} & =\left[\begin{array}{llll}
u_{1} & u_{2} & \ldots & u_{N}
\end{array}\right]^{T} \\
\boldsymbol{v}_{0} & =\left[\begin{array}{llll}
v_{1} & v_{2} & \ldots & v_{N}
\end{array}\right]^{T} \\
\boldsymbol{a}_{0} & =\left[\begin{array}{llll}
a_{1} & a_{2} & \ldots & a_{N}
\end{array}\right]^{T}
\end{aligned}
$$

由式（30）知:

$$
\begin{aligned}
& \boldsymbol{q}_{0((n+m) \times 1)}=\left(\boldsymbol{M}_{P Z}\right)^{-1} \boldsymbol{\Phi}_{Z}^{T} \boldsymbol{M}_{Z} \boldsymbol{u}_{0} \\
& \boldsymbol{q} 1_{0((n+m) \times 1)}=\left(\boldsymbol{M}_{P Z}\right)^{-1} \boldsymbol{\Phi}_{Z}^{T} \boldsymbol{M}_{Z} \boldsymbol{v}_{0} \\
& \boldsymbol{q}_{0((n+m) \times 1)}=\left(\boldsymbol{M}_{P Z}\right)^{-1} \boldsymbol{\Phi}_{Z}^{T} \boldsymbol{M}_{Z} \boldsymbol{\alpha}_{0}
\end{aligned}
$$

这样,便可通过求解式 (34) 得到结构的响应。

在工程实际中, 对于较大型的组合结构, 一般仅关心 前几阶特征对, 所以 $\bar{M}, \bar{K}$ 的维数远小于 $M_{Z}, K_{Z}$ 的维数, 这样经过模态缩减后, 式 (26) 的自由度比式 (23) 的自 由度小得多, 这对实际计算是十分有利的。

对于一般无阻尼 $n$ 自由度线性定常系统，动能和势能 具有如下形式:

$$
T=\frac{1}{2} \dot{\boldsymbol{x}}^{T} \boldsymbol{M} \dot{\boldsymbol{x}}, U=\frac{1}{2} \boldsymbol{x}^{T} \boldsymbol{K} \boldsymbol{x}
$$

又 $\boldsymbol{x}=\boldsymbol{\Phi q}$, 得到:

\begin{tabular}{|c|c|c|c|c|c|c|}
\hline \multirow[b]{2}{*}{ 算例 } & \multicolumn{3}{|l|}{ 基体 } & \multicolumn{3}{|l|}{ 夹杂 } \\
\hline & $\begin{array}{l}E \\
\text { (GPa) }\end{array}$ & $v$ & $\rho\left(\mathrm{kg} / \mathrm{m}^{3}\right)$ & $E(\mathrm{GPa})$ & $v$ & $\rho\left(\mathrm{kg} / \mathrm{m}^{3}\right)$ \\
\hline 材料 & 70 & 0.33 & 2600 & 125 & 0.33 & 8900 \\
\hline
\end{tabular}

$$
\begin{aligned}
& T=\frac{1}{2} \dot{\boldsymbol{q}}^{T} \boldsymbol{M} \dot{\boldsymbol{q}}=\frac{1}{2} \sum_{j=1}^{n} M_{p j} \dot{q}_{j}^{2} \\
& U=\frac{1}{2} \boldsymbol{q}^{T} \boldsymbol{K} \boldsymbol{q}=\frac{1}{2} \sum_{j=1}^{n} K_{p j} q_{j}^{2}
\end{aligned}
$$

对于一个定常系统, 总能量 $W=U+\omega^{2} T$ 为常数。通过 比较不同计算方法的 $\mathrm{W}$ 值的大小来确定计算精度, $\mathrm{W}$ 越小, 精度越高。

\section{4. 数值算例}

为了验证MEM计算结构动力学响应的有效性, 这里 采用一维杆周期结构作为验证模型, 如图2所示, 材料的
属性见表 $1 。 M E M(i)$ 是指结构中加入 $\mathrm{i}$ 个模态进行修正的单 胞模态法, FEM指细观有限元法。

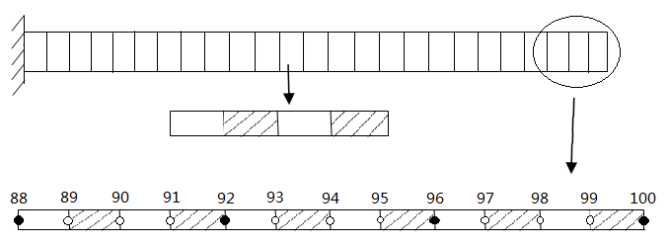

图2 杆结构模型。

表1 材料属性。

图2所示一维梁周期结构由 25 个特征单元组成, 每个 特征单元划分为 4 个细网格, 特征单元单元尺寸为 $1 \mathrm{~mm}$, 细网格有限元中, 单胞中每个小格看作大小为 $0.25 \mathrm{~mm}$ 每 个单胞划分 4 个单元, 结构总细观单元结点数为 100 , 特征 单元结点数为 25 , 结构一边固支一边自由, 自由端施加速 度, 大小为 $1 \mathrm{~m} / \mathrm{s}^{2}$ 。取结构的 100 结点, 98 结点, 94 结点及 90 结点为研究对象, 其中, 100点为特征单元节点, 98、94、 90点是特征单元内部节点。通过数值计算, 得到各点的位 移和速度响应曲线, 如图3 图11所示, 结构总能量值如表2。

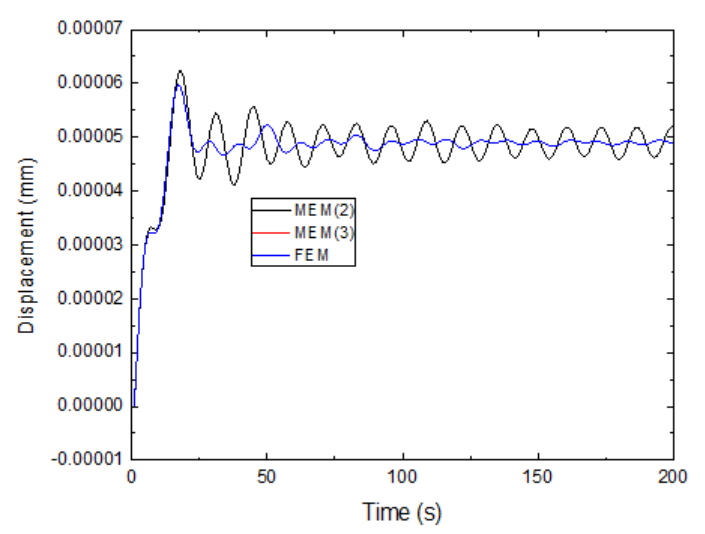

图3 100点位移响应。

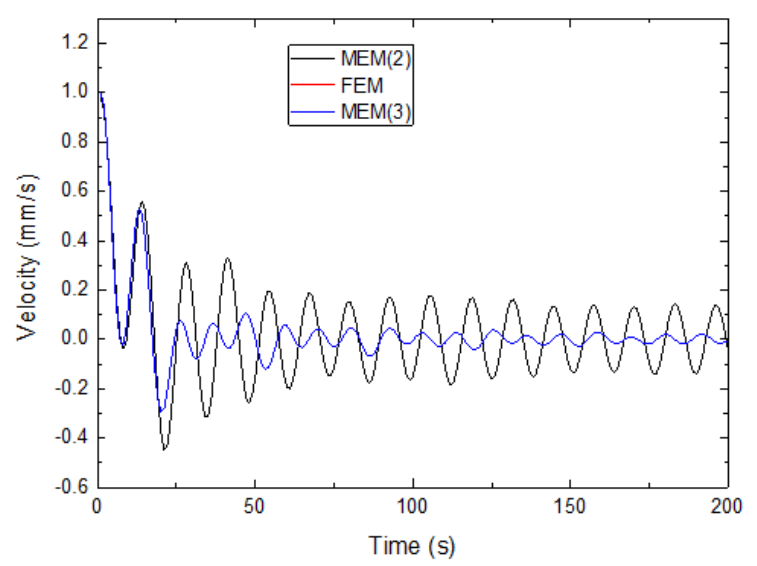

图4 100点速度响应。 


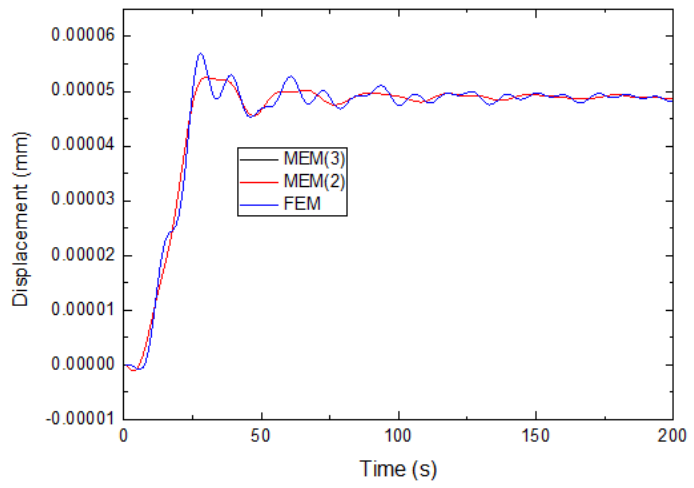

图5 98点位移响应。

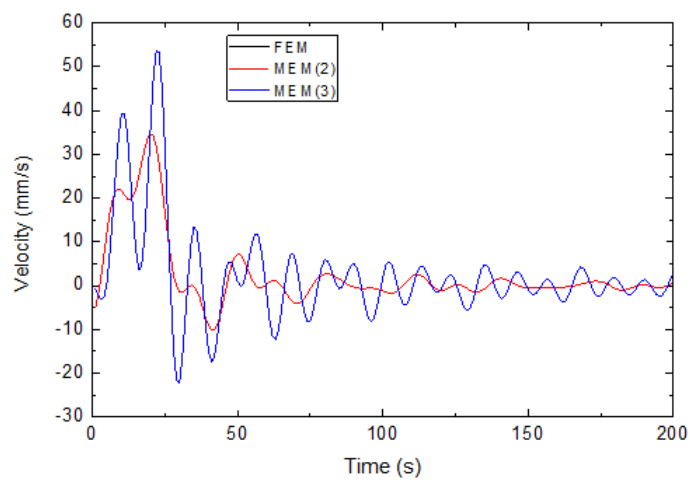

图6 98点速度响应。

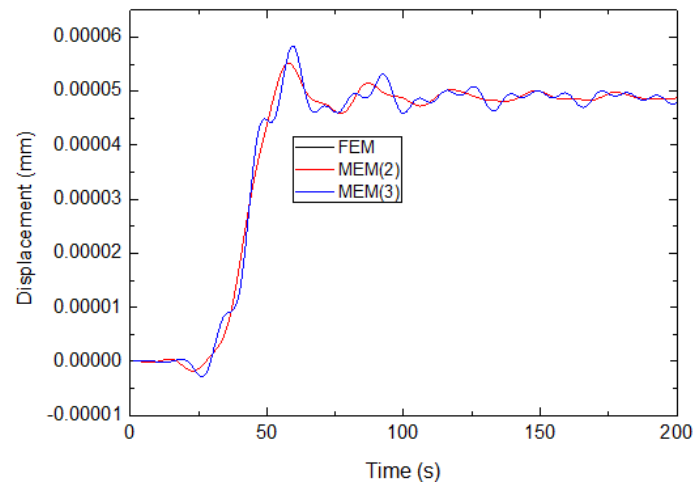

图7 94点位移响应。

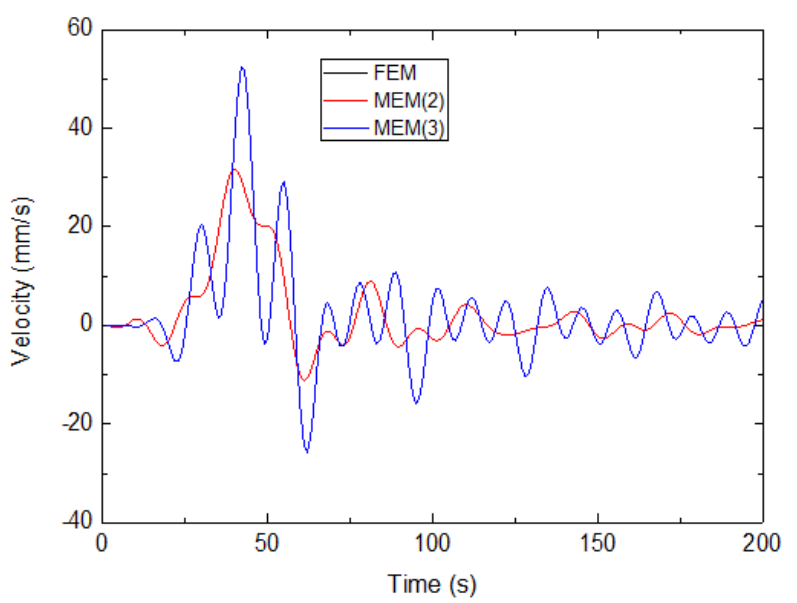

图8 94点速度响应。

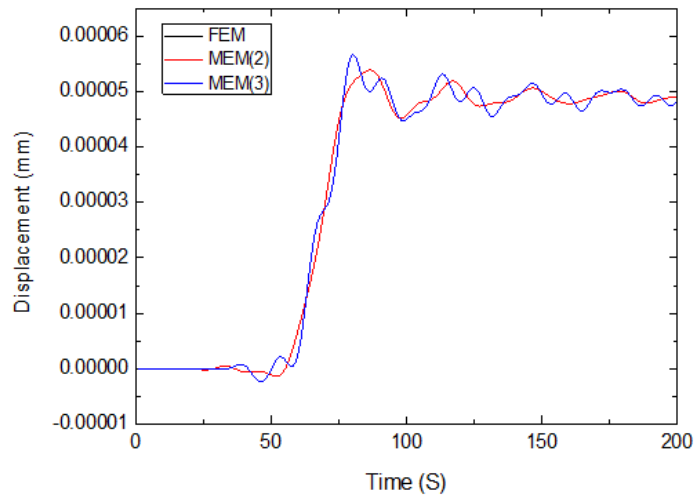

图9 90点位移响应。

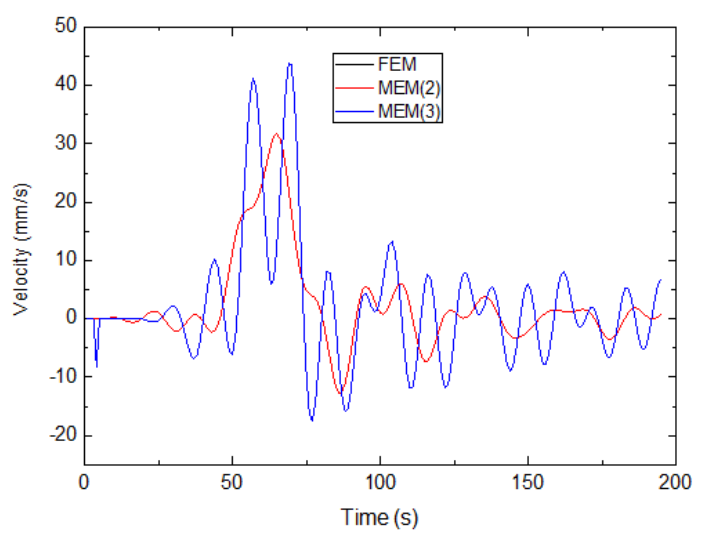

图10 90点速度响应。

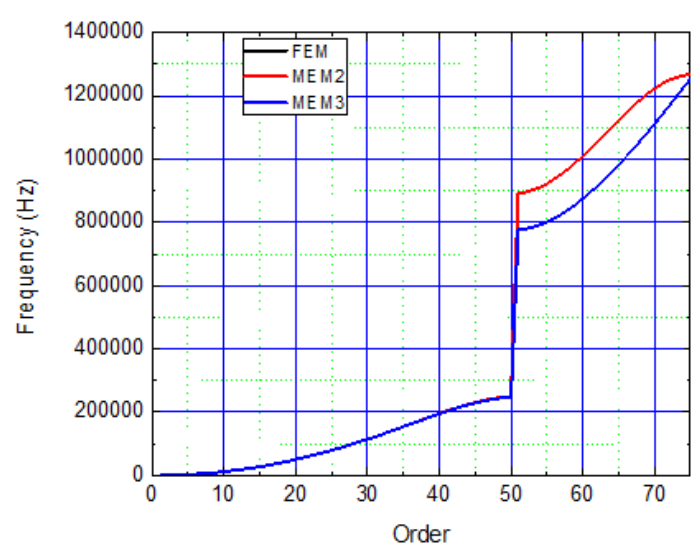

图11 结构频率图。

表2 结构总能量值。

\begin{tabular}{llll}
\hline 算法 & FEM & MEM(3) & MEM(2) \\
\hline$W\left(10^{8}\right)$ & 1.2535 & 1.2535 & 1.2699 \\
$\mathrm{RD}(100 \%)$ & - & 0.0000 & 0.0131 \\
\hline
\end{tabular}

从图3 图11结构响应图中可以看出, MEM加入的模态 修正数越多, 响应与FEM计算结果吻合的越好, 而MEM在保 证精度前提下又有效地降低了计算量, 提高了计算效率。 从结构的频率图, MEM在计算低阶频率有很高的精度, 而 对于一般结构, 其低阶频率是最受关注的, 因此MEM完全 可以满足结构动力学计算精度要求, 由表2可以看出, 当 加入的模态修正数越多, W值越小, 计算精度越高。 


\section{5. 结论}

用一般有限元方法计算时, 各细观单元结构的自由度 相对较小, 易于进行振动特性分析, 当各细观单元结构联 结成整体结构时, 整个结构的自由度有可能较大, 对其进 行振动分析要耗费较多计算时间, 尤其是在结构设计的循 环计算过程中, 需要更多的计算时间, 甚至无法计算。而 用多尺度特征单元方法对结构进行动力学分析时, 引进了 单胞形函数, 由于形函数可以将细观结点与宏观结点联系 起来, 因此用多尺度特征单元方法对结构进行分析时, 由 于形函数的引入巧妙地实现了对复杂结构降维的目的, 大 大降低了结构的自由度, 结构仅包含以各特征单元的主要 振型为坐标的自由度。

本文讨论了多尺度特征单元方法在计算结构动力学 响应的精度特性, 结论和建议如下。（1）梁固有特性的 计算结果表明, 周期复合材料单胞的特征单元在能够反映 单胞几何特性和材料构造细节的前提下, 大幅度减小计算 量。（2）MEM在计算结构动力学响应问题, 其精度取决 于加入的单胞模态数量, 加入的单胞模态数越多, 精度越 高。（3）用最小势能泛函可以有效地评估MEM加入不同 单胞模态数的精度。（4）多尺度特征单元方法计算过程 简便, 也利于编程实现, 并且节约了计算时间, 建议将这 一方法推广到商业领域应用。

\section{参考文献}

[1] Xing YF, Yang Y. An eigenelement method of periodical composite structures. Composite Structures 2011;93:502-512.

[2] Xing YF, Yang Y, Wang XM. A multiscale eigenelement method and its application to periodical compositestructures. Composite Structures 2010; 92:2265-2275.
[3] 邢誉峰, 杨阳. 形函数分段定义的弯矩梁特征单元 [J]. 力学 学报, 2008,40(2):222-228。

[4] 邢誉峰, 田金梅. 三维正交机织复合材料单胞特征单元及其 应用[J]. 航空学报, 2007,28(4):881-885。

[5] 田金梅. 叠层和编织复合材料动态特性研究的新方法 [D]. 北京: 北京航空航天大学, 2005。

[6] Strouboulis T., Babuska I., Copps K. The generalized finite element method: an example of its implementation and illustration of its performance. International Journal for Numerical Methods in Engineering 2000,47:1401-1417.

[7] Xia Z. H., Zhou C. W., Yong Q. L., et al. On selection of repeated unit cell model and application of unified periodic boundary conditions in micro-mechanical analysis of composites [J]. International Journal of Solid and Structures, 2006, 43: 266-278.

[8] Voigt W. Uberdie Beziehung zwischen den beiden Elastizitatskonst-anten isotroper Korper [J]. Wied Ann, 1889, 38: 573-587.

[9] 谢文剑, 诸德超, 邢誉峰. 基于特征向量展开的编织复合材 料的均匀化方法 $[\mathrm{C}]$, 第八届全国振动理论及应用学术会议 论文集，上海，2003年11月。

[10] Y. F. Xing, J. M. Tian, D. C. Zhuand W. J. Xie. The Homogenization Method Based on Eigenvector Expansions for Woven Fabric Composites [J]. International Journal for Multiscale Computational Engineering, 4(1) 197-206 (2006).

[11] Xing Y. F., Du C. Y., An improved multiscale eigenelement method of periodical composite structures [J]. Composite Structures, 2014, 118, 200-207.

[12] 邢誉峰, 高亚贺. 渐进多尺度展开方法的精度和物理意义 [J]. 计算力学学报, 2016,33(4):504-508。

[13] Liu H, Sun X, A hierarchical multilevel finite element method for mechanical analyses of periodical composite structures [J]. Composite Structures, 2015, 131, 115-127. 